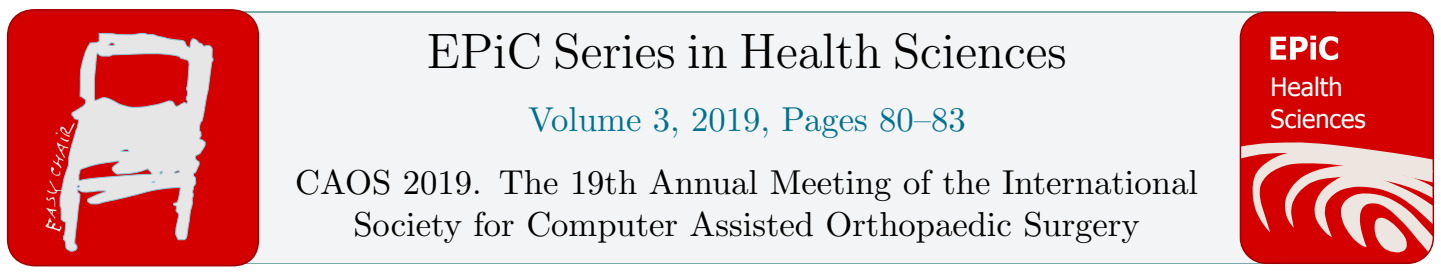

\title{
Early Revision Analysis of Robotic-Arm Assisted and Manual Unicompartmental Knee Arthroplasty
}

\author{
Christina L Cool' ${ }^{1}$; Keith A Needham ${ }^{1}$; Andréa Coppolecchia ${ }^{2}$; Anton Khlopas ${ }^{3}$; \\ Nipun Sodhi ${ }^{4}$, Joseph O. Ehiorobo ${ }^{4}$; Michael A. Mont ${ }^{4}$ \\ ${ }^{1}$ Baker Tilly; ${ }^{2}$ Stryker Corporation; ${ }^{3}$ Cleveland Clinic; ${ }^{4}$ Lenox Hill Hospital, \\ Northwell Health; mmont@,northwell.edu
}

\section{Background:}

Robotic-arm assisted UKA (rUKA) has recently emerged, presenting a technique that could address some of the challenges and intra-operative complications associated with manually-instrumented UKA (mUKA). Most notably, rUKA has demonstrated more accurate component positioning (with significantly lower median errors for all component parameters; $\mathrm{p}<0.01$ ) and a shorter inpatient LOS when compared to mUKA ( 1.7 vs. 2.3 days; $p<0.001)^{1-3}$. The precision and reproducibility of the rUKA technique adds another beneficial dimension over the technically demanding nature of a mUKA ${ }^{4}$. In addition, rUKA has shown lower early post-operative pain, improved patient outcomes, and improved patient satisfaction when compared to mUKA surgeries ${ }^{4-6}$. The clinical differences between rUKA and mUKA, which could impact whether recipients require revision surgery, have economic implications that need to be further evaluated. In response to the evidential gaps in the existing literature, this study seeks to evaluate the real world, national rate of revisions associated with patients undergoing rUKA compared to mUKA procedures in a 24-month postsurgical period. Complementing the clinical value defined in prior studies, the present study also evaluates the costs associated with revision of rUKA compared to mUKA. Specifically, in comparing mUKA to mUKa, we compared: (1) the incidence and characterization of revision procedures; (2) the LOS reduction and costs of the index procedure; and (3) the LOS and costs across the entire 24-month episode of care. 


\section{Methods:}

Data utilized in this analysis were obtained from the OptumInsight Inc. (Eden Prairie, Minnesota) database, comprising claims representing approximately 25 million patients in a U.S. commercial health plan. Patients $\geq 18$ years of age who received either a mUKA or a rUKA procedure were candidates for inclusion and were identified by the presence of appropriate billing codes. Procedures performed between March $1^{\text {st }}, 2013$ and July $31^{\text {st }}, 2015$ were used to calculate the rate of surgical revisions occurring within 24-months of the index procedure. Cases were matched on the following criteria: 1) demographic (age group, gender, and race and comorbid member characteristics (chronic obstructive pulmonary disease (COPD), diabetes, coronary artery disease (CAD), smoking, and obesity); 2) geographic and demographic variations within the United States (using Census Bureau divisions to match on metropolitan areas, rankings of median household income, and college plus education) and 3) concentration of healthcare specialists per 100,000 population. Following propensity matching, 246 rUKA and $492 \mathrm{mUKA}$ patients were included. Revision rates and the associated costs were compared between the two cohorts. The MannWhitney U test, was used to compare continuous variables, and fisher's exact tests was used to analyze discrete categorical variables.

\section{Results:}

Patients who underwent rUKA experienced on average fewer revision procedures $(0.81 \%$ [2/246] vs. 5.28\% [26/492]; $p=0.002)$. These cases experienced shorter hospital stays during their revision procedure ( 2.00 vs. 2.33 days; $p>0.05)$.

During hospitalization for the index procedure, rUKA cases experienced a LOS reduction of $12.38 \%$ ( 1.77 vs. 2.02 days; $p=0.0047$ ) and an associated cost reduction of $2.02 \%$ when compared to mUKA cases ( $\$ 25,786$ vs. $\$ 26,307 ; p>0.05$ ). Table 4 presents the average costs, average LOS, and revision rates for members at index in addition to at 24-months of follow-up.

In addition, rUKA cases experienced a significant LOS reduction of $17.24 \%$ (1.78 vs. 2.15 days $\mathrm{p}=.005$ ) across the entire 24-month inpatient episode of care. Similarly, the mean cost of index plus revisions was $7.06 \%$ less for rUKA when compared to the mUKA cohort (\$26,001 vs. $\$ 27,977 \mathrm{p}>.05)$. 
Table 4: Index and post-index revision rates, average costs, and length of stay by surgery type

\begin{tabular}{|c|l|l|l|l|}
\hline Outcomes Category & rUKA $^{\mathrm{a}}$ & nUKA $^{\mathrm{b}}$ & $\Delta$ & $\mathrm{p}$-Value \\
\hline Total Index Procedures & 246 & 492 & & \\
\hline Index Outcomes & & & & \\
\hline Average Cost & $\$ 25,786$ & $\$ 26,307$ & $-\$ 521$ & 0.3996 \\
\hline Average LOS & 1.77 & 2.02 & -0.25 & 0.0047 \\
\hline 24 Month Revision Outcomes & & & & \\
\hline Rate of Revision [n] & $0.81 \%[2]$ & $\begin{array}{l}5.28 \% \\
{[26]}\end{array}$ & $-4.47 \%$ & 0.0017 \\
\hline Average Cost & $\$ 26,512$ & $\$ 30,430$ & $-\$ 3,918$ & 0.5468 \\
\hline Average LOS & & 2.33 & -0.33 & 0.9277 \\
\hline 24 Month Inpatient Episode & 2.00 & & & \\
\hline Average Cost & $\$ 26,001$ & $\$ 27,977$ & $-\$ 1,975$ & 0.1144 \\
\hline Average LOS & 1.78 & 2.15 & -0.37 & 0.0045 \\
\hline
\end{tabular}

${ }^{a}$ rUKA: Robotic-arm assisted unicompartmental knee arthroplasty

bmUKA: Manual unicompartmental knee arthroplasty

cLOS: Length of Stay

\section{Conclusions:}

In summary, study results indicate that the use of rUKA for the treatment of isolated compartment knee $\mathrm{OA}$ has demonstrated fewer revision surgeries while offering cost savings to the health plan in comparison to mUKA in a 24-month postsurgical period. This study can be used to inform managed care decision-makers on cost containment strategies for the treatment of end-stage knee OA. Moreover, these economic findings supplement the growing body of literature on the clinical utility of rUKA. Beyond these findings, should resource utilization trends continue to show greater shifts towards UKA rather than TKA ${ }^{7,8}$, payers may experience even greater cost reduction.

\section{References}

1. Bell SW, Anthony I, Jones B, MacLean A, Rowe P, Blyth M. Improved Accuracy of Component Positioning with Robotic-Assisted Unicompartmental Knee Arthroplasty: Data from a Prospective, Randomized Controlled Study. $J$ Bone Joint Surg Am United States, 2016;98(8):627-635.

2. Dunbar NJ, Roche MW, Park BH, Branch SH, Conditt MA, Banks SA. Accuracy of dynamic tactile-guided unicompartmental knee arthroplasty. $J$ 
Arthroplasty Elsevier, 2012;27(5):803-8.e1.

3. Plate JF, Mofidi A, Mannava S, Smith BP, Lang JE, Poehling GG, et al. Achieving accurate ligament balancing using robotic-assisted unicompartmental knee arthroplasty. Adv Orthop Hindawi Publishing Corporation, 2013;2013:837167.

4. Kleeblad LJ, Borus TA, Coon TM, Dounchis J, Nguyen JT, Pearle AD. Midterm Survivorship and Patient Satisfaction of Robotic-Arm-Assisted Medial Unicompartmental Knee Arthroplasty: A Multicenter Study. $J$ Arthroplasty United States, 2018;

5. Pearle AD, List JP van der, Lee L, Coon TM, Borus TA, Roche MW. Survivorship and patient satisfaction of robotic-assisted medial unicompartmental knee arthroplasty at a minimum two-year follow-up. Knee Netherlands, 2017;24(2):419-428.

6. Lonner JH. Indications for unicompartmental knee arthroplasty and rationale for robotic arm-assisted technology. Am J Orthop (Belle Mead NJ) 2009;38(2 Suppl):3-6.

7. Moschetti WE, Konopka JF, Rubash HE, Genuario JW. Can RobotAssisted Unicompartmental Knee Arthroplasty Be Cost-Effective? A Markov Decision Analysis. J Arthroplasty 2016;31(4):759-765.

8. Riddle DL, Jiranek WA, McGlynn FJ. Yearly Incidence of Unicompartmental Knee Arthroplasty in the United States. J Arthroplasty 2008;23(3):408-412. 